\title{
PROPUESTA PARA EL DESARROLLO DE CONTENIDOS EN TELEVISIÓN DIGITAL PARA PERSONAS CON LIMITACIÓN AUDITIVA
}

\section{PROPOSAL FOR DEVELOPMENT OF CONTENTS IN DIGITAL TELEVISION FOR HEARING IMPAIRED PEOPLE}

\author{
MSc. Mónica Espinosa Buitrago*, Ing. Ernesto Cadena Muñoz.** \\ Ing. Carlos Montenegro Narvaez*, Ing. Diego Reyes Daza.* \\ *Universidad Santo Tomas, Ingeniería de Telecomunicaciones. \\ Grupo de Investigación INVTEL, USTA.
}

Cra 9, No. 51-11, Bogotá, Cundinamarca, Colombia. Tel.: (+571) 5878797.

E-mail: \{monica.espinosa, carlosmontenegro, diegoreyes\}@usantotomas.edu.co.edu.co.

${ }^{* *}$ SENA, GICS.

Cra. 30, No. 17B-25 Sur, Bogotá, Cundinamarca, Colombia. Tel.: (+571) 5860000.

E-mail: ecadenam@misena.edu.co.

\begin{abstract}
Resumen: En este artículo se ha realizado una caracterización de la población con este tipo de limitación, concluyendo que existen diferentes desarrollos tecnológicos en donde se podrían observar de una forma más interactiva este tipo de contenidos aportando al desarrollo social, y educativo de esta población.
\end{abstract}

Palabras clave: 3D, discapacidad, televisión.

Abstract: In this paper a characterization of the population with this limitation was made, concluding that there are different technological developments which could be observed in an even more interactive way such content that contributes to social and education improvement of this population.

Keywords: 3D, impaired, television.

\section{INTRODUCCIÓN}

En la evolución de los sistemas de televisión se ha buscado generar un impacto a diferentes poblaciones. El objetivo de esta investigación son las personas con limitaciones, por lo cuál se ha desarrollado una caracterización de personas con la limitación de oír aun con aparatos especiales para el diseño tecnológico propuesto. Se inicia con una revisión de la descripción de las personas con limitaciones en Colombia, se definen los actores tales como intérprete de sordo, guía de intérprete y lenguaje de señas, que son de vital importancia en las estrategias existentes tales como texto escondido o closed caption y lenguaje de señas. Se establece que los sistemas o mecanismos que se desarrollen para este propósito serán implementados en televisión digital y se revisa su normatividad en el país. Se analizan las tramas de transporte en televisión y los mecanismos para generar contenidos en 3D que permitan mejorar la interactividad para esta población objetivo.

\section{DESCRIPCIÓN DE PERSONAS CON LIMITACIONES}

Los contenidos son considerados la información transmitida por los sistemas de televisión que permiten a la población establecer un canal de contenidos a nivel social, económico, político y cultural. En el caso Colombiano un porcentaje de la 
población según el censo del departamento de estadística, tienen diferentes tipos de limitaciones y se define como un reto tecnológico ya que se deben establecer plataformas que les permitan un canal de comunicación que se acondicione a su limitación.

De acuerdo con los datos del Censo General del 2005 [2], la tasa de Prevalencia para el total de la población (6.3\%) es mayor en hombres $(6.6 \%)$ que en mujeres $(6.1 \%)$. Teniendo en cuenta el número de limitaciones por persona, el $71.2 \%$ presentan una limitación, el $14.5 \%$ dos limitaciones, el 5.7\% tres limitaciones y el $8.7 \%$ tres o más limitaciones permanentes [2].

Del total de personas que reportaron alguna limitación, el $29.3 \%$ poseen limitaciones para moverse o caminar, el $14.6 \%$ para usar brazos y manos, el $43.4 \%$ para ver a pesar de usar lentes o gafas, el $17.3 \%$ para oír aun con aparatos especiales, el $13.0 \%$ para hablar, el $11.7 \%$ para entender o aprender, 9.4\% para relacionarse con los demás por problemas mentales o emocionales, el 9.9\% para bañarse, vestirse o alimentarse por sí mismo y el 18,8\% presentan otra limitación como se observar en la Tabla 1[2]. Dada esta clasificación, uno de los primeros objetivos que se definen como un reto tecnológico es llegar a la creación de contenidos para la población que tiene la limitación de oír aun con aparatos especiales

\section{Tabla 1. Estadísticas población con limitaciones Fuente: DANE 2005-2006 [2].}

\begin{tabular}{|l|c|}
\hline \multicolumn{1}{|c|}{ Total } & $\begin{array}{c}\text { Porcentaje } \\
\%\end{array}$ \\
\hline Otras limitación permanente & 18,8 \\
\hline $\begin{array}{l}\text { Bañarse, vestirse, alimentarse por sí } \\
\text { mismo }\end{array}$ & 9,5 \\
\hline Relacionarse con los demás & 9,9 \\
\hline Entender o Aprender & 12,1 \\
\hline Hablar & 13,0 \\
\hline Oír, aun con aparatos especiales & 17,3 \\
\hline Ver, a pesar de usar lentes o gafas & 43,4 \\
\hline Usar brazos o manos & 14,6 \\
\hline Moverse o caminar & 29,3 \\
\hline
\end{tabular}

Para este fin, se caracterizó de acuerdo al marco normativo colombiano ley 982 de 2005 [3] la definición de las variables que se deben tener en un sistema para realizar la interpretación adecuada para esta limitación.

- Intérprete para sordos: Persona con amplios conocimientos de la Lengua de Señas
Colombiana que puede realizar interpretación simultánea del español hablado en la Lengua de Señas y viceversa. También son intérpretes para sordos aquellas personas que realicen la interpretación simultánea del castellano hablado a otras formas de comunicación de la población sorda, distintas a la Lengua de Señas, y viceversa. [3].

- Guía intérprete: Persona que realiza una labor de transmisión de información visual adaptada, auditiva o táctil, descripción visual del ambiente en donde se encuentre y guía en la movilidad de la persona sordociega, con amplio conocimiento de los sistemas de comunicación que requieren las personas sordociegas [3].

- La Lengua de Señas: en Colombia la utilizan quienes no pueden desarrollar lenguaje oral, se entiende y se acepta como idioma necesario de comunicación de las personas con pérdidas profundas de audición y las sordociegas, que no pueden consiguientemente por la gravedad de la lesión desarrollar lenguaje oral, necesarios para el desarrollo del pensamiento y de la inteligencia de la persona, por lo que debe ser reconocida por el Estado y fortalecida por la lectura y la escritura del castellano, convirtiéndolos propositivamente en bilingüales [3].

Basados en el marco normativo colombiano, se realiza una revisión de las estrategias existentes que permiten establecer contenidos para esta población, dando como resultado dos opciones:

\subsection{Texto escondido o Closed Caption}

Es el proceso en el cual se convierte el audio de un programa de televisión, transmisión de internet, película, video y demás producciones, en línea de texto, este aparecerá en la pantalla o monitor. Es importante diferenciar entre subtítulos y closed caption, son la traducción de las palabras de los contenidos pero sin identificar el hablante, por el contrario el Closed Caption diferencia entre las personas que interactúan en la conversación y además se describe los efectos de sonido y música.

Existen leyes en cada país que estandarizan este tipo de contenidos, en Colombia la ley 982 del 2 de agosto de 2005[3], establece las obligaciones de los productores de contenidos de televisión hacia la población con limitación de oír aun con aparatos especiales. El Closed Caption debe ser preciso, consistente, claro, fácil de leer y equivalente. 


\subsection{Lenguaje de Señas}

Es el proceso mediante el cual se integra a un contenido audiovisual una imagen en el cual un personaje traduce en vivo al lenguaje de señas usado por las personas con discapacidad auditiva.

En la actualidad existen acuerdos y leyes que normalizan el uso de este lenguaje para cada uno de los contenidos. En Colombia el acuerdo del año 2012 [4], se especifica los deberes que tiene los canales públicos en el acceso a sus contenidos para las personas con esta limitación, observando la necesidad de este contenido en nuestro país.

\section{TELEVISIÓN DIGITAL}

En el Acuerdo 001 de la Comisión Nacional [4]. Los sistemas que permiten el acceso al servicio público de televisión a las personas sordas e hipoacústicas son [4]:

- Interpretación en Lengua de Señas Colombiana (LSC).

- Texto escondido o Closed Caption (CC) por sus siglas en idioma inglés.

- Subtitulación (ST).

- Los sistemas o mecanismos que se desarrollen con posterioridad para este propósito.

Para la televisión digital en el artículo 7 se podrán utilizar los siguientes mecanismos [4]:

- Interpretación en Lengua de Señas (LSC).

- Subtitulación.

- Los sistemas o mecanismos que se desarrollen con posterioridad para este propósito.

Desde un punto de vista técnico, la información transmitida en los sistemas de televisión digital requiere ser multiplexada en tramas de transporte.

Dichas tramas contienen señales de audio, video y datos que deben ser enviadas por un solo canal de transmisión. La interactividad del usuario puede ser relacionada con los datos como se muestra en la figura 1 [5].

Las señales de televisión tienen contenidos de video en estándares HD (High Definition), SD (Standard Definition) y UHD (Ultra High Definition).

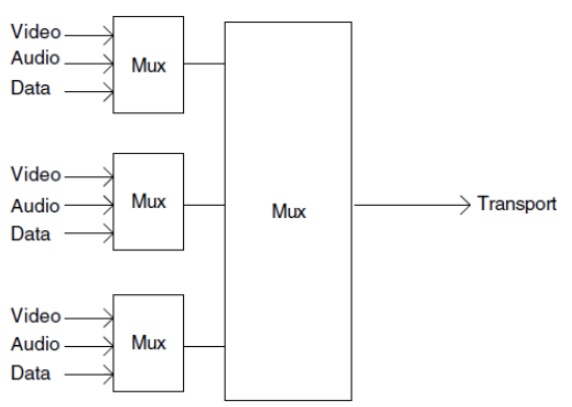

Fig. 1. Tramas de Transporte.

Fuente: Fundamentos de televisión [5].

En la optimización del canal de transmisión de la información, la televisión digital debe realizar un procesamiento que permita mejorar las tasas de transmisión como se observa en la figura 2 [5]

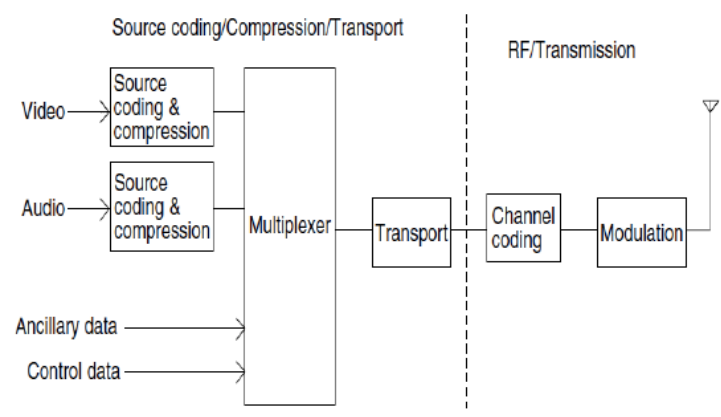

Fig. 2. Procesamiento digital de televisión. Fuente: Fundamentos de televisión [5].

Estos modelos tradicionales de procesamiento de las señales, no determinan cómo la interactividad debe ser procesada en la tramas de transporte (TS). Para ello se debe realizar un estudio que permita evaluar su comportamiento frente a las aplicaciones que han surgido tales como: televisión sobre el protocolo de internet (IPTV), satelital, terrestre y móvil.

Por otra parte, la interactividad en los sistemas de televisión digital (TDi) ha sido considerada un factor muy importante en el desarrollo de los contenidos para el usuario final, ya que permite interactuar sincrónica y asincrónicamente con los mismos. De forma sincrónica el usuario tendrá un canal para la subida y bajada de contenidos que le permitirá interactuar en tiempo real, lo que sería contrario a nivel de tiempos para los sistemas asincrónicos.

$\mathrm{Al}$ establecer contenidos comunicativos las brechas digitales disminuyen [6]. Por ello en la propuesta se establecen TS que soporten contenido de imágenes en tercera dimensión (3D) para personas con limitación auditiva. 


\subsection{Procesamiento de imágenes en tercera dimensión (3D)}

Actualmente, los sistemas de captura de movimiento tienen aplicaciones en campos como salud, física, ingeniería y arquitectura. Se ha logrado la captura de objetos como una araña [7]. El análisis de sus movimientos recrea un modelo mecánico que simula su movimiento [8], como se puede ver en la figura 3 [9].
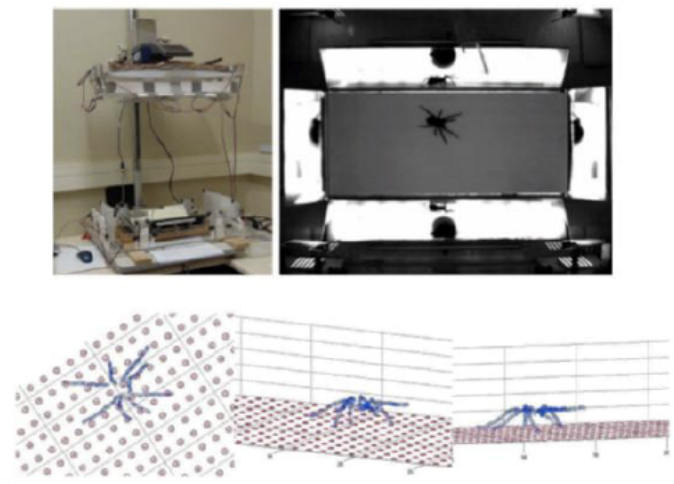

Fig. 3. Aplicación de captura de animales. Fuente: The Design and Calibration of a 3D Motion Capture System for Arthropods [7].

En la robótica, se puede copiar o simular estos movimientos para conseguir robots más flexibles y robustos [10] [11]. En la salud, permite obtener información sobre la postura y movimientos de un paciente, descubriendo problemas y permitiendo un diagnostico y tratamiento a pacientes más sencillo, como se observa en la figura $4[1]$.

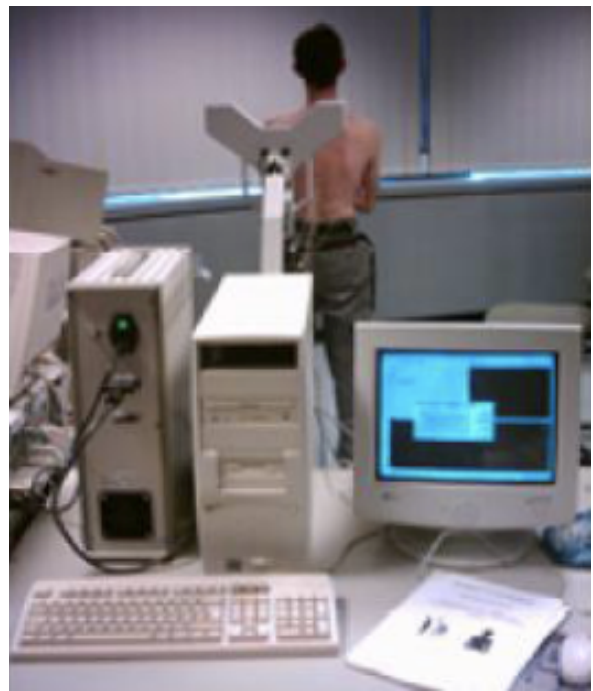

Fig. 4. Aplicación en el movimiento de las personas.

Fuente: 3D Motion Capture Methods for Pathological and Nonpathological Human Motion Analysis [1].
Se presentan avances en el tema de prevención de cáncer con algoritmos que permiten descubrir la aparición de cáncer de seno, poder tratarlo adecuadamente y a tiempo, este desarrollo puede observarse en la figura 5 [12].
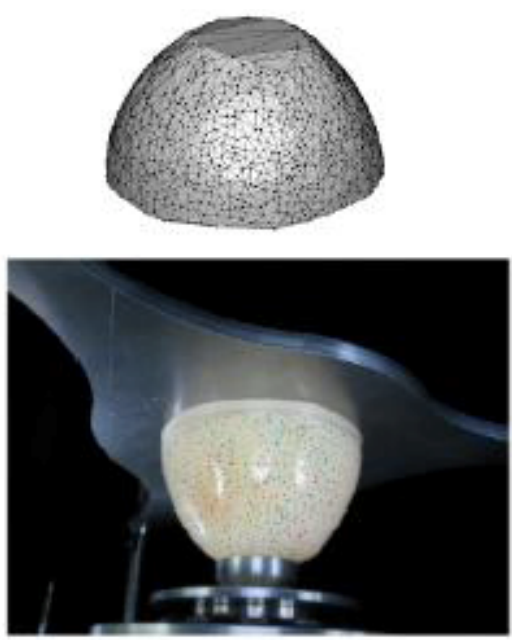

Fig. 5. Aplicación en prevención de cáncer. Fuente: Vision-based 3D surface motion capture for the DIET breast cancer screening system [12].

El reconocimiento de los movimientos del cuerpo ha sido explorado en varias áreas, específicamente el movimiento de los brazos ha sido capturado para señales de vuelo [13]. Se usaron señales que utilizan los árbitros en baloncesto, como se observa en la figura 6 [13].
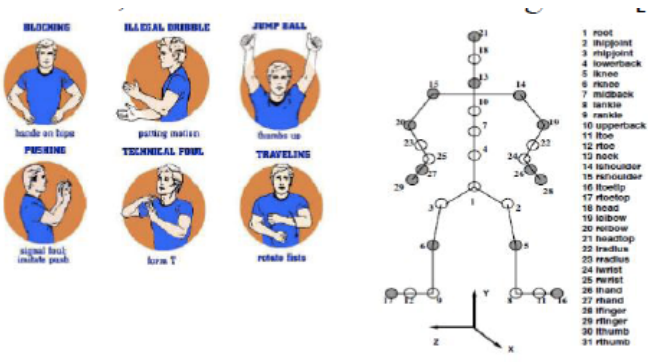

Fig. 6. Reconocimiento de movimientos del cuerpo. Fuente: Handsignals recognition from video using $3 D$ motion capture data [13].

A una escala más pequeña, se encuentran aplicaciones de captura facial que han evolucionado a sistemas que permiten la captura con cámaras de alta resolución y modelos como Active Appearance Models (AAM), el resultado es mejor, mas natural y humano [14][15][16]. En la figura 7 [14] se observa un test de captura facial. En la figura 8 [14] se observa la generación del modelo y captura facial para juegos en 3D. 
Revista Colombiana de Tecnologías de Avanzada

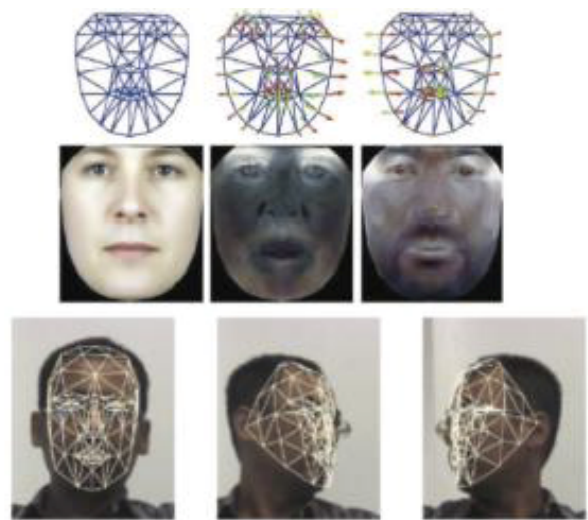

Fig. 7. Test de Captura Facial.

Fuente: Facial motion capture with $3 D$ active appearance models [14].
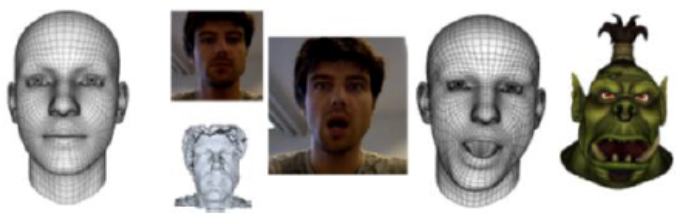

Fig. 8. Captura Facial para animación en 3D. Fuente: Facial motion capture with 3D [14].

Capturar el movimiento de las manos es otra de las aplicaciones desarrolladas [17], como se observa en la Figura 10 [17]. En la Figura 9 [16] se muestran algunos modelos de la captura de manos [16], donde se puede ver la mano articulada, las partes conectadas o numeradas.

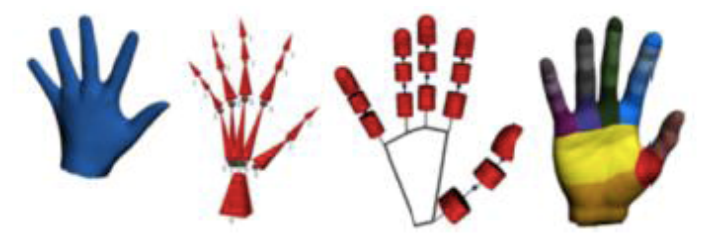

Fig. 9. Modelos de estimación de la pose de mano. Fuente: A Survey on Human Motion [16].

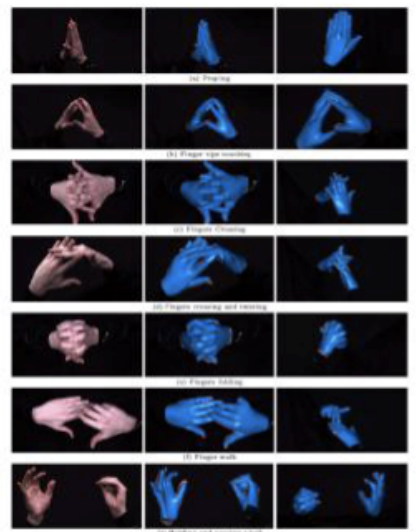

Fig. 10. Captura de movimientos de la mano. Fuente: Motion capture of hands in action [17].

\section{PROPUESTA PARA EL DESARROLLO DE CONTENIDOS EN TELEVISIÓN DIGITAL PARA PERSONAS CON LIMITACIÓN AUDITIVA}

$\mathrm{Al}$ establecer la población con limitación auditiva como objeto de esta propuesta y teniendo en cuenta los requerimientos de la ley, en donde se aclara que se podrían desarrollar propuesta futura para abarcar la interactividad en los contenidos de televisión para las personas con esta discapacidad se define la siguiente metodología para el desarrollo de esta propuesta:

Fase 1: Diseño de la infraestructura de telecomunicaciones para sistema de televisión digital interactiva. Se dividirá en el procesamiento de señal de televisión y del sistema para medir las variables de la televisión. En la Tabla 2 se observan los equipos de esta etapa. $\mathrm{Al}$ contar con el módulo DT504 se manejarán contenidos de televisión en SD (Standard Definition), que serán convertidos a tipo ASI (Interface serial Asincrónica) y empaquetados en TS como se observa en la Figura 11. Las TS podrán ser transmitidas en diferentes formatos con los módulos DT102 de televisión digital terrestre y el DT421D televisión en IP (Internet Protocol).

Tabla 2 Equipos Fase de procesamiento de señal.

\begin{tabular}{|l|l|l|}
\hline Módulo & $\begin{array}{l}\text { Fabricante/E } \\
\text { ntidad }\end{array}$ & Descripción \\
\hline DT8000 & $\begin{array}{l}\text { PROMAX/SE } \\
\text { NA }\end{array}$ & $\begin{array}{l}\text { Fuente de Poder los } \\
\text { modulos }\end{array}$ \\
\hline DT102 & $\begin{array}{l}\text { PROMAX/SE } \\
\text { NA }\end{array}$ & $\begin{array}{l}\text { Modulador DVB T } \\
\text { Doble }\end{array}$ \\
\hline DT421D & $\begin{array}{l}\text { PROMAX/SE } \\
\text { NA }\end{array}$ & Conversor ASI a IP \\
\hline DT504B & $\begin{array}{l}\text { PROMAX/SE } \\
\text { NA }\end{array}$ & $\begin{array}{l}\text { Codificador de } \\
\text { video/audio }\end{array}$ \\
\hline $\begin{array}{l}\text { Icareus } \\
\text { iTV } \\
\text { Suite }\end{array}$ & ICAREUS/SE & $\begin{array}{l}\text { Software para } \\
\text { aplicaciones } \\
\text { interactivas. }\end{array}$ \\
\hline
\end{tabular}

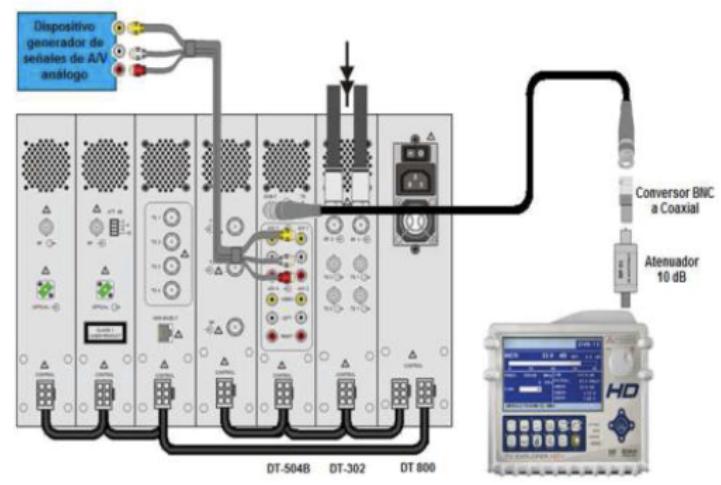

Fig. 11. Propuesta gráfica del diseño. Fuente: PROMAX. 
Al emitir estos contenidos se analizará la emisión y las TS por medio de los equipos que se observan en la Tabla 3 que abarcan la fase de instrumentación del sistema de televisión.

Tabla 3: Equipos Fase de procesamiento de señal.

\begin{tabular}{|l|l|l|}
\hline Módulos & Fabricante/Entidad & Descripción \\
\hline $\begin{array}{l}\text { TV } \\
\text { Explorer }\end{array}$ & PROMAX/SENA & $\begin{array}{l}\text { Analizador } \\
\text { de redes de } \\
\text { televisión }\end{array}$ \\
\hline $\begin{array}{l}\text { ETL TV } \\
\text { Analyzer }\end{array}$ & $\begin{array}{l}\text { R\&S/Universidad } \\
\text { Santo Tomás }\end{array}$ & $\begin{array}{l}\text { Analizador } \\
\text { de tramas de } \\
\text { televisión. }\end{array}$ \\
\hline
\end{tabular}

Fase 2: Diseño de los contenidos de televisión en 3D compatible con los sistemas de televisión.

A nivel comercial, sistemas de captura como el impulsex2 de Phasespaces Lab [9], permiten captura en tiempo real, recreando modelos solidos de los datos capturados, con aplicaciones que llegan a empresas como la NASA, Boeing, Lockheed Martin y Disney. Dentro de las aplicaciones desarrolladas encontramos las animaciones para la televisión como se observa en la figura 12 [18]. Además, de los reconocidos juegos dance central 3 y spiderman, como se puede ver en la Figura 13 [18] .

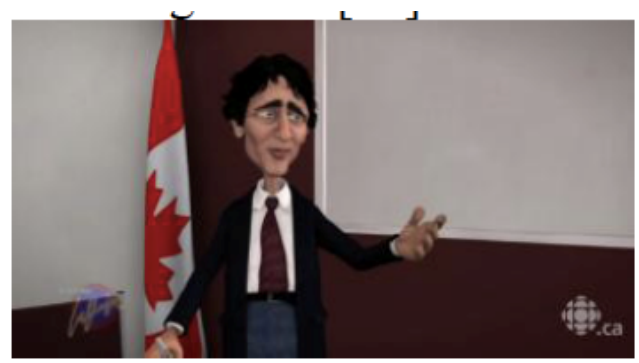

Fig. 12. Animación para televisión. Fuente: http://laflaque.radio-canada.ca/ [18].

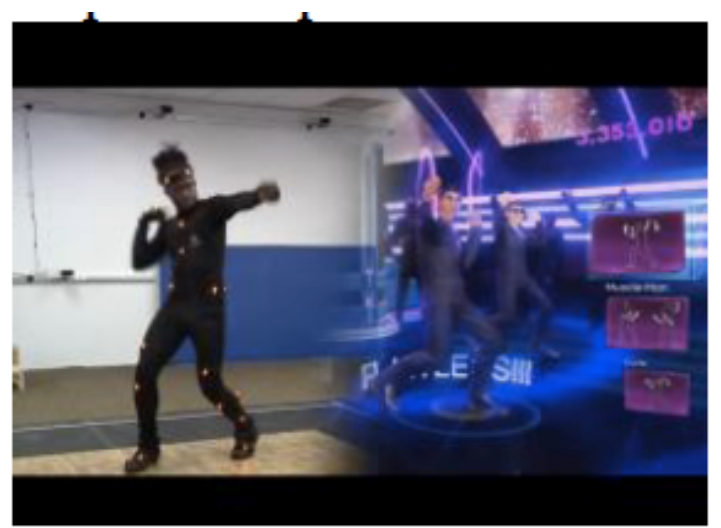

Fig. 13. Animación para Dance Central. Fuente: http://laflaque.radio-canada.ca/ [18]
Para este proceso de captura y animación se utilizan los equipos que se pueden observar en la Tabla 4.

\section{Tabla 4 Equipos Fase de captura de movimientos.}

\begin{tabular}{|c|c|c|}
\hline Módulos & $\begin{array}{l}\text { Fabricante/ } \\
\text { Entidad }\end{array}$ & Descripción \\
\hline CAMARAS & $\begin{array}{l}\text { PHASESPA } \\
\text { CE/SENA }\end{array}$ & $\begin{array}{l}\text { Cámara de alta } \\
\text { resolución. }\end{array}$ \\
\hline $\begin{array}{l}\text { CONTROL } \\
\text { ADORES } \\
\text { DE LED }\end{array}$ & $\begin{array}{l}\text { PHASESPA } \\
\text { CE/SENA }\end{array}$ & $\begin{array}{l}\text { Controladores de } \\
\text { LEDs }\end{array}$ \\
\hline $\begin{array}{l}\text { LEDS } \\
\text { ACTIVOS }\end{array}$ & $\begin{array}{l}\text { PHASESPA } \\
\text { CE/SENA }\end{array}$ & $\begin{array}{l}\text { Leds activos de luz } \\
\text { roja o infraroja }\end{array}$ \\
\hline SERVIDOR & $\begin{array}{l}\text { PHASESPA } \\
\text { CE/SENA }\end{array}$ & $\begin{array}{ll}\text { Servidor } & \text { de } \\
\text { Información } & \text { de } \\
\text { captura. } & \\
\end{array}$ \\
\hline $\begin{array}{l}\text { CALIBRAC } \\
\text { ION } \\
\text { TUBO DE }\end{array}$ & $\begin{array}{l}\text { PHASESPA } \\
\text { CE/SENA }\end{array}$ & $\begin{array}{lr}\text { Sirve para la } \\
\text { calibración del } \\
\text { sistema de captura. }\end{array}$ \\
\hline
\end{tabular}

Fase 3: Los sistemas o mecanismos que se desarrollen con posterioridad para este propósito.

\section{CONCLUSIONES}

Se desarrolló un estudio de clasificación de limitaciones en Colombia que nos permitió elegir la población objeto de estudio que son las personas con limitación de oír aun con aparatos especiales, encontrando variables importantes en el marco legal Colombia que nos permiten definir y delimitar los actores y los alcances de un diseño tecnológico para televisión.

Se estudiaron los mecanismos que permiten un canal de comunicaciones para dicha población, según la normatividad y se concluye que para televisión digital se podrán implementar "los sistemas o mecanismos que se desarrollen con posterioridad para este propósito" en el caso de personas sordas e hipoacústicas.

Se revisó el funcionamiento de televisión por medio de tramas de transporte para incluir un lenguaje de señas capturado en un sistema de 3D y esta manera incluir contenidos interactivos que permitan a esta población un mejor nivel cultural y educativo.

Se define la propuesta de desarrollar contenidos interactivos en 3D, a partir de la captura de movimientos, insertados en tramas TS de televisión digital, para convertir información de texto en animaciones digitales y beneficiar la población con limitación auditiva en general. 


\section{AGRADECIMIENTOS}

Universidad Santo Tomás, Facultad de Ingeniería de Telecomunicaciones, Bogotá. También a los grupos de investigación INVTEL, USTA y GICS del SENA. Y por último a Tecnoparque SENA, Bogotá

\section{REFERENCIAS}

[1]. DANE. Dirección de Censos y Demografía, "Base de Datos del Registro de Localización y Caracterización de Personas con Discapacidad." 2006-2005.

[2]. El Congreso de Colombia, "Ley 982 de 2005," Agosto-2005.

[3]. Comisión Nacional de Televisión, "Acuerdo 01", 2012.

[4]. G. W. Collins, Fundamentals of digital television transmission, vol. 1. Wiley, 2001.

[5]. A. Garcia-Crespo, B. Ruiz-Mezcua, I. Gonzalez-Carrasco, J. L. Lopez-Cuadrado, Z. Hernandez, R. Barahona, and L. H. de Toppin, "Accessibility Services and Interactive Digital Television" Tecnol. Aprendiz. IEEE Rev. Iberoam. De, vol. 9, no. 1, pp. 8-16, 2014.

[6]. T. J. Hoermann, S. Mills, M. Paulin, and S. Reusenzehn, "The design and calibration of a 3D motion capture system for arthropods," in (IVCNZ), 2013 28th International Conference of, 2013, pp. 265-269.

[7]. S. Reuls senzehn, "Mechanical design of the legs of Dolomedes aquaticus-Novel approaches to quantify the hydraulic contribution to joint," University of Otago, 2010.

[8]. Phasespace, "ImpulseProductDoc." 2014.

[9]. M. Brambilla, E. Ferrante, M. Birattari, and M. Dorigo, "Swarm robotics: a review from the swarm engineering perspective," Swarm Intell., vol. 7, no. 1, pp. 1-41, 2013.
[10]. M. F. Silva and J. T. Machado, "A literature review on the optimization of legged robots," J. Vib. Control, vol. 18, no. 12, pp. 17531767, 2012.

[11]. Z. Kertesz and I. Lovanyi, "3D Motion Capture Methods for Human Motion Analysis," in Information and Communication Technologies, 2006. ICTTA'06. 2nd, vol. 1, pp. 1062-1067.

[12]. R. G. Brown, C. E. Hann, and J. G. Chase, "Vision-based 3D surface motion capture for the DIET breast cancer screening system," Int. J. Comput. Appl. Technol., vol. 39, no. 1, pp. 72-78, 2010.

[13]. T. P. Tian and S. Sclaroff, "Handsignals recognition from video using $3 \mathrm{D}$ motion capture data," in Application of Computer Vision, 2005. WACV/MOTIONS'05 Volume 1. Seventh IEEE Workshops on, 2005, vol. 2, pp. 189-194.

[14]. C. Darujati and M. Hariadi, "Facial motion capture with 3D active appearance models," in Instrumentation, Communications, (ICICI$B M E), 2013$, pp. 59-64.

[15]. M. Hossny, D. Filippidis, W. Abdelrahman, H. Zhou, M. Fielding, J. Mullins, L. Wei, D. Creighton, V. Puri, and S. Nahavandi, "Low cost multimodal facial recognition via kinect sensors," in LWC 2012:Proceedings of the 2012 Land Warfare Conference, 2012, pp. 77-86.

[16]. T. B. Moeslund, A. Hilton, and V. Krüger, "A survey of advances in vision-based human motion capture and analysis," Comput. Vis. Image Underst., vol. 104, no. 2-3, pp. 90 - 126, 2006.

[17]. L. Ballan, A. Taneja, J. Gall, L. Van Gool, and M. Pollefeys, "Motion capture of hands in action using discriminative salient points," in Computer Vision-ECCV 2012, Springer, 2012, pp. 640-653.

[18]. aflaque.radio-canada, "http://laflaque.radiocanada.ca/." 2014 